
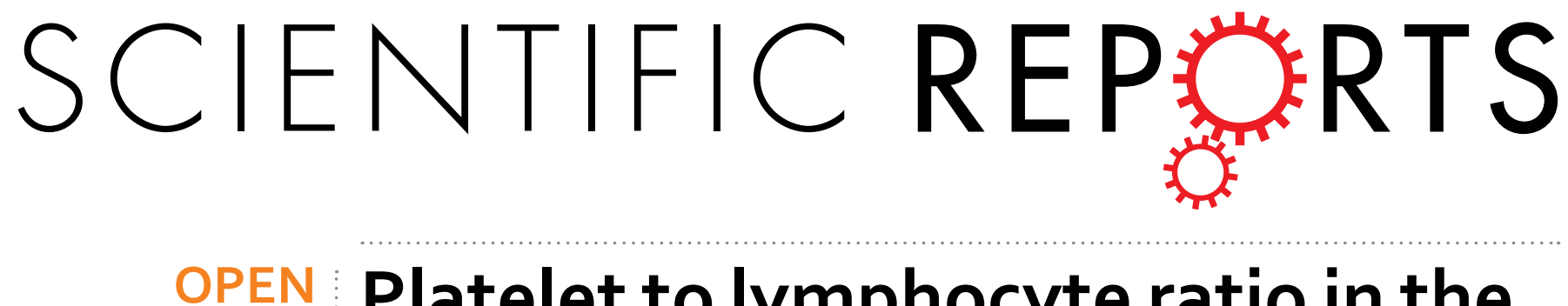

\title{
Platelet to lymphocyte ratio in the prediction of adverse outcomes after acute coronary syndrome: a
}

Received: 08 June 2016

Accepted: 05 December 2016

Published: 10 January 2017

\section{meta-analysis}

\author{
Wenzhang $\mathrm{Li}^{1, *}$, Qianqian $\mathrm{Liu}^{2, *}$ \& Yin Tang ${ }^{3}$
}

Recent studies have shown platelet to lymphocyte ratio (PLR) to be a potential inflammatory marker in cardiovascular diseases. We performed a meta-analysis to systematically evaluate the prognostic role of PLR in acute coronary syndrome (ACS). A comprehensive literature search up to May 18, 2016 was conducted from PUBMED, EMBASE and Web of science to identify related studies. The risk ratio (RR) with $95 \%$ confidence interval $(\mathrm{Cl})$ was extracted or calculated for effect estimates. Totally ten studies involving 8932 patients diagnosed with ACS were included in our research. We demonstrated that patients with higher PLR level had significantly higher risk of in-hospital adverse outcomes $(R R=2.24$, $95 \% \mathrm{Cl}=1.81-2.77)$ and long-term adverse outcomes $(\mathrm{RR}=2.32,95 \% \mathrm{Cl}=1.64-3.28)$. Sensitivity analyses confirmed the stability of our results. We didn't detect significant publication bias by Begg's and Egger's test $(p>0.05$ ). In conclusion, our meta-analysis revealed that PLR is promising biomarker in predicting worse prognosis in ACS patients. The results should be validated by future large-scale, standard investigations.

Acute coronary syndrome (ACS), characterized by unstable atherosclerotic lesions, is the main cause of death from coronary heart diseases ${ }^{1}$. Other major adverse cardiovascular event (MACE) following ACS, such as re-infarction and recurrent ischaemia, also pose heavy burden on health-care resources ${ }^{2}$. Even with the advent of coronary intervention techniques and progress in medication, the prognosis of ACS is still not satisfactory ${ }^{3}$. Thus, it is of great significance to identify high-risk ACS patients who require more intensive control of risk factors, more aggressive therapy and more close follow-up.

The interaction of inflammation and thrombosis is the critical factor in the pathogenesis of $\mathrm{ACS}^{4}$. As a reflection of excess inflammatory status and thrombotic activity, elevated peripheral blood platelet count is regarded to be a valuable predictor of adverse cardiovascular outcomes. And multiple researches have demonstrated this speculation $^{5,6}$. On the other hand, decreased lymphocyte count is also related to worse cardiovascular prognosis, which may be partly explained by the role of lymphocyte in protection of plaque stability ${ }^{7}$. In this context, a novel marker, platelet to lymphocyte ratio (PLR), seemed to be a potential indicator in ACS prognosis.

Recently, the prognostic importance of PLR has been investigated by several studies. Zhou et al. demonstrated that PLR was positively associated with the Global Registry of Acute Coronary Events (GRACE) risk score and can improve its the predictive power for long-term cardiovascular events in patients with $\mathrm{ACS}^{8}$. Temiz et al. reported that PLR was an independent predictor of in-hospital cardiovascular mortality in patients with ST-elevated acute myocardial infarction ${ }^{9}$. Ugur et al. found that there was a significant association between high PLR levels and the adjusted risk of 6-month all-cause deaths in ST elevation myocardial infarction underwent primary coronary intervention, while with no difference in in-hospital mortality among patients with different PLR levels ${ }^{10}$. Although most studies demonstrated the positive association between PRL and ACS adverse outcomes, there are still some discrepancies. Hence, we performed this meta-analysis to investigate the role of PLR in

\footnotetext{
${ }^{1}$ Department of Cardiology, First Affiliated Hospital of Chengdu Medical College, Chengdu, Sichuan, China. ${ }^{2}$ Department of Respiratory Diseases, Chengdu Municipal First People's Hospital, Chengdu, Sichuan, China. ${ }^{3}$ State Key Laboratory of Oral Disease, West China School \& Hospital of Stomotology, Sichuan University, Chengdu, Sichuan, China. *These authors contributed equally to this work. Correspondence and requests for materials should be addressed to Q.L. (email: chuan112500@126.com)
} 


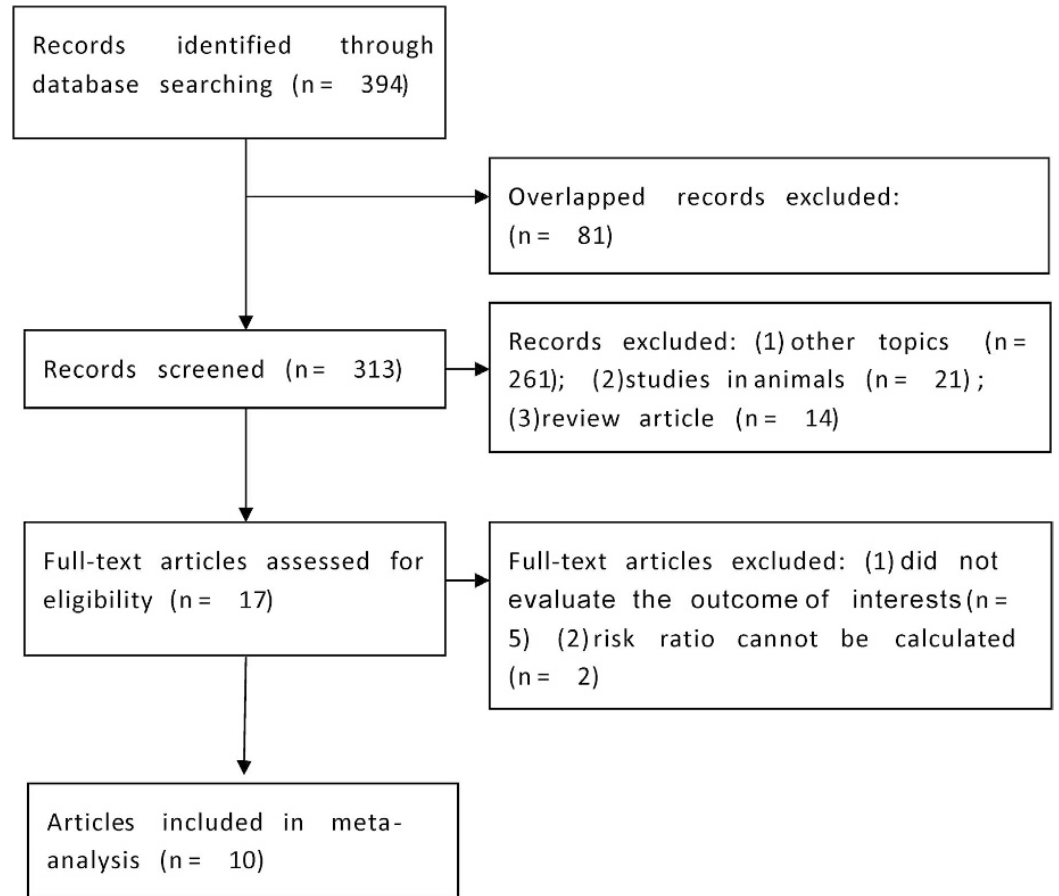

Figure 1. Flow diagram of included studies.

evaluating the prognosis of ACS, focusing on in-hospital and long-term adverse outcomes respectively. This study was reported according to the Preferred Reporting Items for Systematic Reviews and Meta-Analyses (PRISMA) ${ }^{11}$.

\section{Methods}

Search strategy. We conducted a comprehensive computer search through the following databases from their inception until May 18, 2016: PUBMED, EMBASE and Web of Science. No language restriction was applied. The following terms was used to identify related articles: 'platelet' in combination with 'lymphocyte', and in combination with 'acute coronary syndrome' or 'myocardial infarction' or 'myocardial ischaemia' or 'unstable angina'. The reference lists of all included studies were also screened to check for relevant articles.

Inclusion and exclusion criteria. The inclusion criteria were listed as follows: (1) All patients were diagnosed as ACS, including acute ST elevation myocardial infarction, acute non-ST elevation myocardial infarction and unstable angina ${ }^{8}$. (2) Blood samples for PLR determination were taken at admission. (3) Outcomes were death, re-infarction, postinfarction angina, recurrent ischaemia or need for revascularization ${ }^{12}$. Patients were followed up during hospitalization (in-hospital outcomes) or for at least six month (long-term outcomes) (4) Sufficient data were reported to estimate the relative risk (RR) with $95 \%$ confidence interval (CI). Exclusion criterion was duplicates of previous publications.

Data extraction and quality assessment. Two investigators (Qianqian Liu and Wenzhang Li) were responsible for data extraction and quality assessment. The following information were obtained: title, journal, author, language, year of publication, study design, country, sample size, mean age, gender, diagnosis, outcomes, adjusted or unadjusted risk estimates. The Newcastle-Ottawa scale (NOS) was used to assess the methodological quality of included studies, which is composed of three aspects (selection, comparability and outcome), with 9 being the highest score. We considered studies with a NOS score more than or equal to seven to be of high quality. Discrepancies were discussed by the two reviewers.

Statistical analysis. We performed meta-analysis on association between PLR and ACS prognosis using RR estimates and 95\% CIs on a natural logarithmic scale. Compared with crude RR, the adjusted RR were preferred when available. We transformed adjusted odds ratio (OR) to RR when necessary with methods provided by Zhang et al. ${ }^{13}$. Statistical heterogeneity was tested by Cochran's $Q$ statistic and $\mathrm{I}^{2}$ tests. Fixed effect model was used if the $p>0.10$ and $\mathrm{I}^{2}<50 \%$ otherwise the random effect model was adopted ${ }^{14,15}$. In order to confirm the robust of pooled results, we performed a sensitivity analysis by excluding each study one by one. Begg's and Egger's tests were conducted to identify possible publication bias ${ }^{16}$. Statistical analyses were done with STATA version 12.0 (StataCorp, College Station, Texas), using metan program. $P$ values were two-sided.

\section{Results}

Study characteristics. Totally ten studies involving 8932 patients diagnosed with ACS were included in our

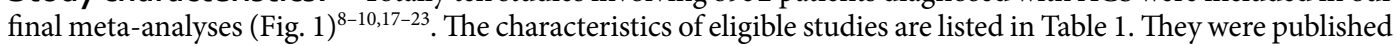
between 2012 and 2016, and conducted at hospitals from Europe (seven in Turkey, one in Poland), Asia (one in 


\begin{tabular}{|c|c|c|c|c|c|c|c|c|c|c|c|c|}
\hline $\begin{array}{l}\text { Author } \\
\text { (year) }\end{array}$ & Study design & Country & $\begin{array}{l}\text { Sample } \\
\text { size }\end{array}$ & $\begin{array}{c}\text { Mean age } \\
\text { (years) }\end{array}$ & $\begin{array}{c}\text { Male } \\
(\%)\end{array}$ & Diagnosis & $\begin{array}{c}\text { Adverse } \\
\text { outcomes }\end{array}$ & $\mathbf{R R}^{\mathbf{a}}$ & $\mathbf{R R}^{\mathbf{b}}$ & $\begin{array}{c}\text { Mean follow-up } \\
\text { (months) }\end{array}$ & Cut-off & Nos \\
\hline Ugur $^{10}$ & prospective cohort & Turkey & 639 & 56 & 84.80 & STEMI & Death & $\begin{array}{c}\mathrm{RR}=0.55 \\
(0.15-1.93)\end{array}$ & $\begin{array}{c}\mathrm{RR}=2.37 \\
(1.06-4.97)^{\mathrm{d}}\end{array}$ & 6 & 174.9 & 8 \\
\hline Toprak $^{17}$ & prospective cohort & Turkey & 304 & 60 & 80.90 & STEMI & MACE & $\begin{array}{c}\mathrm{RR}=2.27 \\
(1.04-4.97)\end{array}$ & $\begin{array}{c}\mathrm{RR}=2.62 \\
(1.44-4.77)\end{array}$ & 24 & 141 & 7 \\
\hline Zhou $^{8}$ & prospective cohort & China & 2230 & 59 & 58.10 & ACS & MACE & NA & $\begin{array}{c}\mathrm{RR}=1.56 \\
(1.36-1.80)\end{array}$ & 72 & 170 & 7 \\
\hline Temiz $^{9}$ & retrospective cohort & Turkey & 636 & 62 & 82.50 & STEMI & Death & $\begin{array}{c}\mathrm{RR}=2.16 \\
(1.16-4.0)^{\mathrm{c}}\end{array}$ & NA & NA & 144 & 9 \\
\hline Cetin $^{16}$ & prospective cohort & Turkey & 1938 & 60 & 66.40 & STEMI & MACE & $\begin{array}{c}\mathrm{RR}=2.43 \\
(1.53-3.88)\end{array}$ & $\begin{array}{c}\mathrm{RR}=2.81 \\
(2.01-3.92)\end{array}$ & 31.6 & NA & 8 \\
\hline Oylumlu ${ }^{19}$ & retrospective cohort & Turkey & 587 & 62 & 68.40 & ACS & Death & $\begin{array}{c}\mathrm{RR}=3.05 \\
(1.75-5.29)\end{array}$ & NA & NA & NA & 8 \\
\hline Kurtul $^{20}$ & prospective cohort & Turkey & 1016 & 61 & 71.90 & ACS & Death & $\begin{array}{c}\mathrm{RR}=3.08 \\
(1.70-5.58)\end{array}$ & NA & NA & 116 & 7 \\
\hline Hudzik $^{21}$ & prospective cohort & Poland & 523 & 64 & 41.50 & STEMI & Death & $\begin{array}{c}\mathrm{RR}=1.59 \\
(1.03-2.46)\end{array}$ & $\begin{array}{c}\mathrm{RR}=4.08 \\
(2.72-6.12)\end{array}$ & 12 & 124 & 7 \\
\hline $\mathrm{Azab}^{22}$ & prospective cohort & USA & 619 & $61-68$ & 68.50 & NSTEMI & Death & NA & $\begin{array}{c}\mathrm{RR}=1.62 \\
(1.15-2.26)^{\mathrm{e}}\end{array}$ & 48 & 176 & 8 \\
\hline $\mathrm{Ayca}^{23}$ & retrospective cohort & Turkey & 440 & $56-59$ & 66.80 & AMI & Death & $\begin{array}{c}\mathrm{RR}=3.28 \\
(1.34-8.06)\end{array}$ & NA & NA & 137 & 8 \\
\hline
\end{tabular}

Table 1. Characteristics of studies included in meta-analysis. Abbreviations: RR, risk ratio; NOS, Newcastle-Ottawa scale; STEMI, ST elevated myocardial infarction; MACE: non-fatal major adverse cardiovasculara events; ACS, acute coronary syndrome; NA: not available; NSTEMI, non-ST elevated myocardial infarction; AMI, acute myocardial infarction; PLR: platelet to lymphocyte ratio; GRE, glomerular filtration rate; TIMI, thrombolysis in mycocardial infarction. ${ }^{a} \mathrm{RR}$ of PLR on in-hospital adverse outcomes. ${ }^{\mathrm{b}} \mathrm{RR}$ of PLR on long-term adverse outcomes (more than one month). 'Age, no thromolytic treatment, GFR. ${ }^{\mathrm{c}}$ Age, sex, hypertension, left ventricular ejection fraction, anemia, post TMID flow, Killip class, GFR, three-vessel disease. ${ }^{e}$ GRACE score, use of aspirin or clopidogrel, prior coronary bypass surgery, diabetes mellitus, use of statin, end stage renal disease, prior cerebrovascular events.

China), and America (one in USA) respectively. The sample size of each study ranged from 304 to 2230 . The cutoff value for PLR ranged from 116 to 174.9 in eight studies. While the other two studies didn't present specific cut-off values and they divided patients into groups according to the PLR tertiles (comparing the third tertile versus others). The follow-up duration of long-term adverse outcomes was between one to sex years. Among all these studies, seven were prospective cohort, while the other three were retrospective cohort. All of ten studies were classified to be high-quality, according to NOS score.

Data synthesis. Eight studies, including 6083 patients were evaluated for in-hospital adverse outcomes while six study, involving 6253 patients were assessed for long-term adverse outcomes. The between-study heterogeneity was insignificant in in-hospital adverse outcomes $(p=0.180)$, while obvious in long-term adverse outcomes $(p<0.001)$.

Compared with lower PLR level, patients with higher PLR level had significantly higher risk of in-hospital adverse outcomes $(\mathrm{RR}=2.24 ; 95 \% \mathrm{CI}=1.81-2.77)$, see Fig. 2 . The subsequent subgroup analyses based on different mean age, sample size, region, outcome and confounding factors adjustment were presented in Table 2, which demonstrated the robust poor prognosis among patients with higher PLR level $(p<0.05)$. Sensitivity analysis was shown in Fig. 3. After excluding each study one by one, the corresponding pooled RRs were not obviously changed.

The pooled RRs of long-term adverse outcomes were 2.32 (95\%CI: 1.64-3.28) for patients with higher PLR level, see Fig. 4. The risk effect of higher PLR level on long-term adverse outcomes was not significantly influenced by age, sample size, region, outcome and confounding factors adjustment $(\mathrm{p}<0.05)$, as is shown in Table 2 . It is worth mentioning that the between-study heterogeneity disappeared during the subgroup analyses according to region, which may partly account for the source of heterogeneity. To examine the influence of individual study upon the results, sensitivity analysis was conducted and we arrived almost the same results (Fig. 5).

Publication bias. Begg's funnel plot and Egger's test were performed to evaluate the publication bias of the included studies. The funnel plot was symmetric for both in-hospital and long-term adverse outcomes (Figs 6 and 7). Both Begg's and Egger's test showed lack of publication bias $(p=0.662$ and $p=0.121)$.

\section{Discussion}

PLR, as a systematic inflammatory response indicator, was initially introduced into clinical practice to improve the prognosis prediction of oncologic disorders, such as periampullary cancer, esophageal cancer and gastric cancer et al. ${ }^{24-27}$. After that this marker was found to be associated with inflammation in cardiovascular diseases and positively correlated with SYNTAX score, GRACE risk and no-reflow phenomenon in ACS9 ${ }^{20,28,29}$. Most recently 


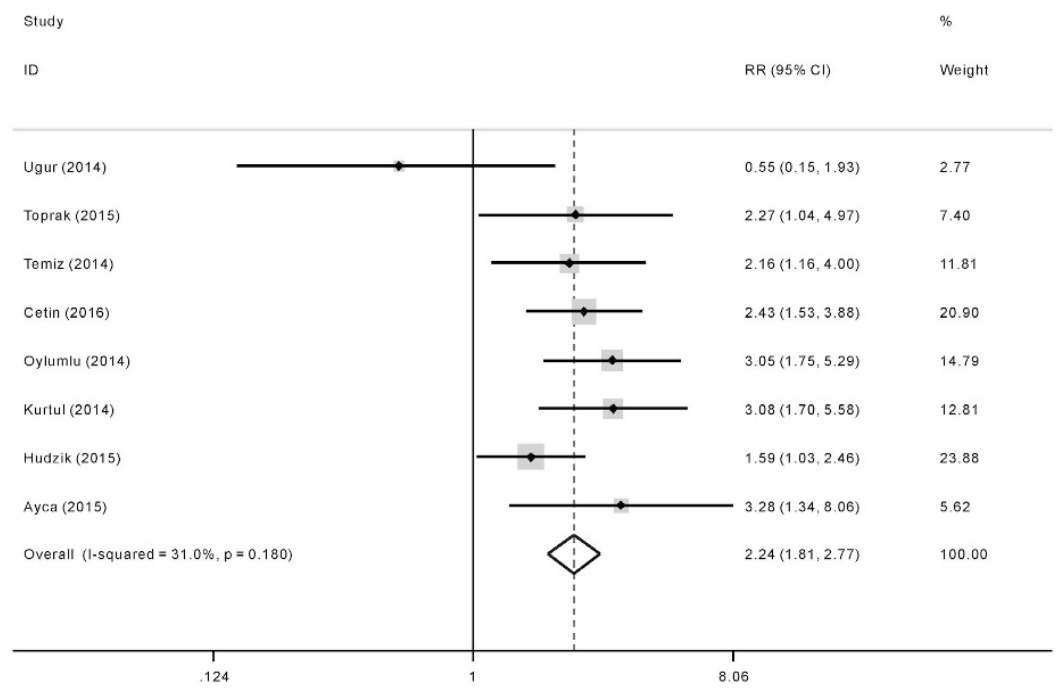

Figure 2. Forrest plot of risk ratio (RR) for the association of platelet to lymphocyte ratio (PLR) with inhospital adverse outcomes after acute coronary syndrome (ACS).

a series of studies have investigated the predictive importance of PLR in the adverse outcomes among ACS and reported inconsistent results ${ }^{9,10,22,29}$. We conducted this meta-analysis to comprehensively understand the prognostic role of PLR in ACS. Our results demonstrated that patients with higher PLR level had 2.24-fold higher risk of in-hospital adverse outcomes and 2.32-fold higher risk of long-term adverse outcomes. The reliability and stability of our results were confirmed by publication bias and sensitivity analyses.

The between-study heterogeneity was insignificant when analyzing in-hospital adverse outcomes, which indicated the reliability of our pooling results. However, there was obvious heterogeneity when pooling the data regarding PLR and long-term adverse outcomes. Then we did subgroup analyses to search for potential factors that lead to heterogeneity. Our subgroup analyses demonstrated that the risk effects of higher PLR level in ACS adverse outcomes were not significantly influenced by potential confounding factors, such as age, sample size, region, outcome and risk factors adjustment. It should be pointed out that the between-study heterogeneity disappeared during the subgroup analyses according to region. We postulate that region may partly explained the observed heterogeneity and it might be ascribed to the following factors. First, as genetic variants have already be demonstrated to be related to hematological traits based on a series of genetic association studies, we believe that the predictive significance of PLR may also be different because of ethnicitic genotypic diversity ${ }^{30}$. And PLR also showed significant ethnic variability in other diseases, such as non-small cell lung cancer ${ }^{31}$. Second, some environmental factors, such as season variation, dietary habit and so on, which were reported to be confounding factors of ACS prognosis may also account for different results from different regional areas ${ }^{32,33}$. However those are only speculations before more evidences were obtained.

The underlying mechanisms of the association between higher PLR and worse prognosis seem to be multifactorial. Firstly, higher platelet counts serve to be both a result and a precipitating factor of inflammatory response. It is reported that megakaryocyte could be stimulated by several inflammatory mediators and presented accelerated proliferation and platelet-production ${ }^{34}$. On the other hand, platelets can release thromboxanes and other mediators, promote the adhesion and transmigration of monocytes, which may cause increased inflammation and weakened plaque stability, then promote the progression of atherosclerosis ${ }^{35-38}$. Additionally the procoagulant role of platelets in the process of homeostasis and thrombosis may also contribute to the progression of the arterial thrombi ${ }^{39}$. On the contrary, the elevated lymphocyte in ischaemic and reperfused myocardium can regulate mononuclear cell phenotype transformation and induce tissue inhibitor of matrix metalloproteinase-1 production, thereby increasing plaque stability in ACS patients ${ }^{7}$. Beyond that lymphopenia was speculated to represent depressed immune response to excess cortisol production during physiological stress, which may lead to worse clinical outcomes ${ }^{40}$. And it has been reported that lymphocytopenia was independently related to adverse cardiac events, advanced heart failure, as well as reduced hemodynamics and aerobic capacity ${ }^{41-45}$. Taken all above together, as a combined marker of thrombocytosis and lymphocytopenia, PLR may be promising in ACS prognosis prediction.

The significance of PLR in ACS prognosis prediction can also be validated through its association with other predictors. Ayhan et al. found that patients with higher PLR levels exhibited higher peak CK-MB, creatinine, Killip class, admission glucose, and anemia in their study ${ }^{46-48}$. Zhou et al. showed a positive association between GRACE risk score and $\mathrm{PLR}^{8}$. The PLR was also reported to be significantly correlated with other inflammatory markers such as C-reactive protein (CRP) and fibrinogen, which have been demonstrated to have predictive and prognostic significance in cardiovascular diseases ${ }^{39}$. Up till now, we didn't find systematic research comparing the significance of those predictors. Which independent or joint predictor is the best of choice is still a matter of debate. However, with easy and rapid availability and relative low costs, PLR is undoubtedly a promising marker which need to be further explored. 


\begin{tabular}{|c|c|c|c|c|c|c|c|c|c|c|c|}
\hline \multirow[b]{2}{*}{ Subgroup } & \multirow[b]{2}{*}{ parameter } & \multicolumn{5}{|c|}{ in-hospital adverse outcomes } & \multicolumn{5}{|c|}{ long-term adverse outcomes } \\
\hline & & $\begin{array}{c}\text { No. of } \\
\text { studies }\end{array}$ & $\begin{array}{c}\text { No. of } \\
\text { patients }\end{array}$ & $\mathrm{RR}(95 \% \mathrm{CI})$ & $p$ value & $\begin{array}{c}p \text { for } \\
\text { heterogeneity }\end{array}$ & $\begin{array}{c}\text { No.of } \\
\text { studies }\end{array}$ & $\begin{array}{c}\text { No. of } \\
\text { patients }\end{array}$ & $\mathrm{RR}(95 \% \mathrm{CI})$ & $p$ value & $\begin{array}{c}p \text { for } \\
\text { heterogeneity }\end{array}$ \\
\hline \multirow{2}{*}{ mean age } & $\leq 60$ & 4 & 3321 & $\begin{array}{c}2.24 \\
(1.58-3.19)\end{array}$ & $<0.001$ & 0.141 & 4 & 5111 & $\begin{array}{c}2.19 \\
(1.47-3.26)\end{array}$ & $<0.001$ & 0.005 \\
\hline & $>60$ & 4 & 2762 & $\begin{array}{c}2.24 \\
(1.72-2.93)\end{array}$ & $<0.001$ & 0.196 & 2 & 1142 & $\begin{array}{c}2.55 \\
(1.03-6.31)\end{array}$ & 0.042 & 0.001 \\
\hline \multirow{2}{*}{ sample Size } & $\leq 500$ & 2 & 744 & $\begin{array}{c}2.66 \\
(1.48-4.80)\end{array}$ & 0.001 & 0.094 & 1 & 304 & $\begin{array}{c}2.62 \\
(1.44-4.77)\end{array}$ & 0.002 & $\mathrm{NA}$ \\
\hline & $>500$ & 6 & 5339 & $\begin{array}{c}2.19 \\
(1.74-2.74)\end{array}$ & $<0.001$ & 0.544 & 5 & 5949 & $\begin{array}{c}2.28 \\
(1.55-3.35)\end{array}$ & $<0.001$ & $<0.001$ \\
\hline \multirow{2}{*}{ region } & Europe & 8 & 6083 & $\begin{array}{c}2.24 \\
(1.81-2.77)\end{array}$ & $<0.001$ & 0.180 & 4 & 3404 & $\begin{array}{c}3.08 \\
(2.46-3.86)\end{array}$ & $<0.001$ & 0.414 \\
\hline & non-Europe & 0 & 0 & NA & $\mathrm{NA}$ & $\mathrm{NA}$ & 2 & 2849 & $\begin{array}{c}1.57 \\
(1.38-1.79)\end{array}$ & $<0.001$ & 0.840 \\
\hline \multirow{2}{*}{ outcomes } & Death & 6 & 3841 & $\begin{array}{c}2.19 \\
(1.70-2.81)\end{array}$ & $<0.001$ & 0.075 & 3 & 1781 & $\begin{array}{c}2.50 \\
(1.30-4.81)\end{array}$ & 0.006 & 0.003 \\
\hline & MACE & 2 & 2242 & $\begin{array}{c}2.39 \\
(1.60-3.56)\end{array}$ & $<0.001$ & 0.883 & 3 & 4472 & $\begin{array}{c}2.17 \\
(1.37-3.45)\end{array}$ & 0.001 & 0.002 \\
\hline \multirow{2}{*}{ adjustment } & yes & 1 & 636 & $\begin{array}{c}2.16 \\
(1.16-4.01)\end{array}$ & 0.015 & $\mathrm{NA}$ & 2 & 1258 & $\begin{array}{c}1.72 \\
(1.26-2.35)\end{array}$ & 0.001 & 0.376 \\
\hline & no & 7 & 5447 & $\begin{array}{c}2.25 \\
(1.80-2.83)\end{array}$ & $<0.001$ & 0.119 & 4 & 4995 & $\begin{array}{c}1.87 \\
(1.66-2.11)\end{array}$ & $<0.001$ & $<0.001$ \\
\hline
\end{tabular}

Table 2. Subgroup analysis. Abbreviations: RR, risk ratio; NOS; Newcastle-Ottawa scale; STEMI, ST elevated myocardial infarction; MACE: non-fatal major adverse cardiovascular events; ACS, acute coronary syndrome; NA: not available; NSTEMI, non-ST elevated myocardial infarction; AMI, acute myocardial infarction; PLR: platelet to lymphocyte ratio; GRE, glomerular filtration rate; TIMI, thrombolysis in mycocardial infarction.

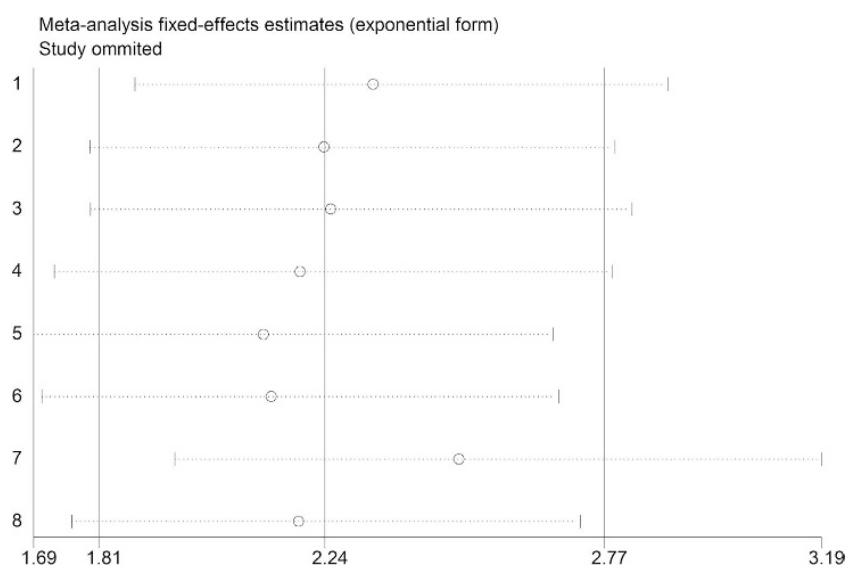

Figure 3. Sensitivity analysis of risk ratio (RR) for the association of platelet to lymphocyte ratio (PLR) with in-hospital adverse outcomes after acute coronary syndrome (ACS).

It is interesting that, in some cases, thrombocytopenia may also indicate increased risk of ischemic events, which seems to be a paradox to our results ${ }^{49,50}$. However, further studies found that, in the population of ischemic patients, it was the use of intra-aortic balloon pump (IABP), but not thrombocytopenia per se, serves as a possible primary cause of adverse ischemic events ${ }^{49,50}$. It reminds us that when we use PLR to predict the prognosis of ACS, we should make our judgment according to each specific matter considering possible confounding factors.

We had to admit that although there is good reason for PLR to be used as a predictive markers in worse prognosis among ACS patients, our research didn't provide direct evidence of independent association between them. Other blood cell counts parameters, which are accompanied by or interfere with the change of PLR, were also demonstrated to be associated with adverse cardiovascular outcomes. For example, elevated red blood cell count, which is usually accompanied with activation, adhesiveness, and aggregation of platelet, can bring about increased blood viscosity and then further promote the genesis of ischaemic heart disease ${ }^{51}$. Puddu et al. reported that increased red blood cell count is independently associated with risk of cardiovascular atherosclerotic events in their study of 6 years follow-up ${ }^{52}$. On the other hand, anemia were also a concomitant of platelet activation in ACS patients as the consequence of chronic inflammatory response ${ }^{53}$. Several researches reported anemia was related to death as well as MACE and which was inferred to be the result of oxygen supply and demand imbalance, rennin-angiotensin-aldosterone activation and sypathetic nervous system activation ${ }^{53-55}$. In a word, whether or not PLR serves as the causal factor for adverse outcomes in ACS patients need to be verified. 


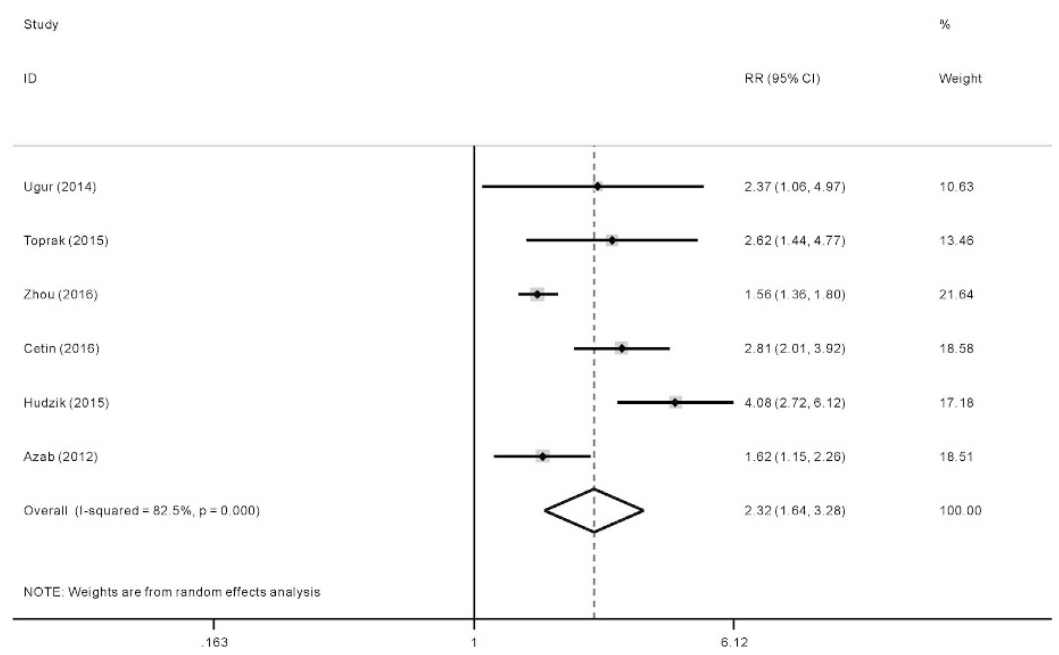

Figure 4. Forrest plot of risk ratio (RR) for the association of platelet to lymphocyte ratio (PLR) with longterm adverse outcomes after acute coronary syndrome (ACS).

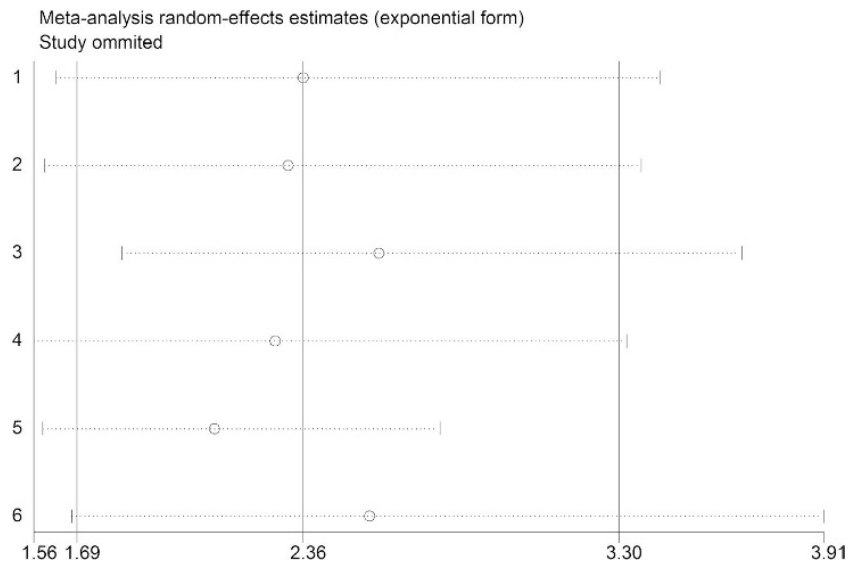

Figure 5. Sensitivity analysis of risk ratio (RR) for the association of platelet to lymphocyte ratio (PLR) with long-term adverse outcomes after acute coronary syndrome (ACS).

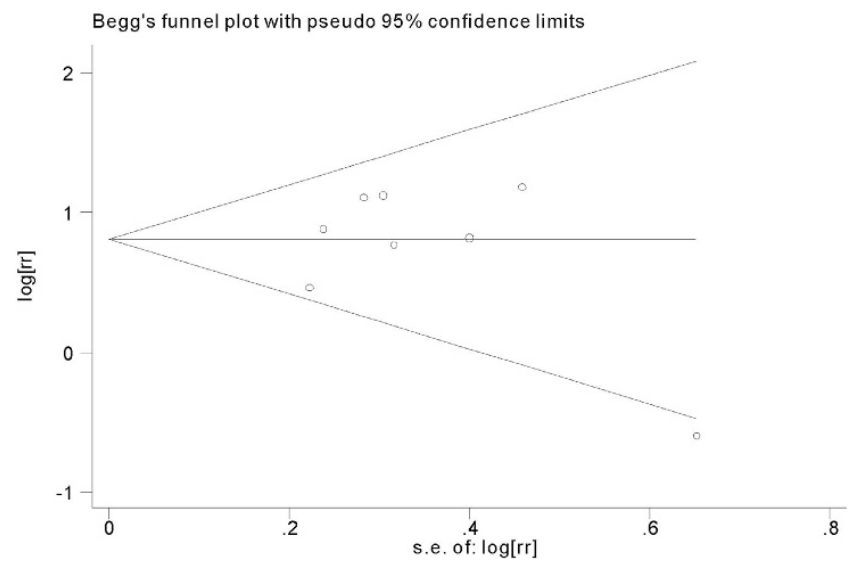

Figure 6. Funnel plot of selected studies for the association of platelet to lymphocyte ratio (PLR) with in hospital adverse outcomes after acute coronary syndrome (ACS).

Some limitations in our meta-analysis should be addressed. First of all, most studies included in our research were conducted in European countries, which may weaken the representative of results. Secondly, some studies did not adjust for potential confounders, which may limit the accuracy of the risk estimates. Thirdly, the cut-off 


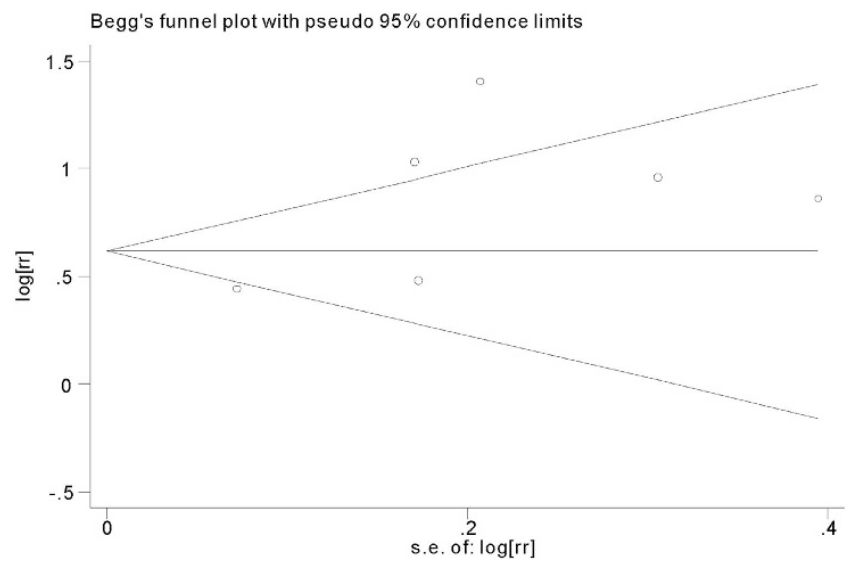

Figure 7. Funnel plot of selected studies for the association of platelet to lymphocyte ratio (PLR) with longterm adverse outcomes after acute coronary syndrome (ACS).

point of PLR in two studies was not described, which limit the application of our research. Finally, some statistical limitations in our research should also be pointed out. No matter we use Egger's or Begg's test, we had to mention that the power to detect publication bias is low with a small number of included studies ${ }^{56}$. What's more, Cochran's $Q$ and $I^{2}$, which were adopted to detect between-study heterogeneity in our study, can be misleading in meta-analyses if the appropriate reference $Q$ value is not considered ${ }^{57}$.

In conclusion, our meta-analysis revealed that PLR is promising biomarker in predicting both in-hospital and long-term worse prognosis in ACS patients. The results should be validated by future large-scale, standard investigations.

\section{References}

1. Writing Group, M. et al. Heart Disease and Stroke Statistics-2016 Update: A Report From the American Heart Association. Circulation 133, e38-60, doi: 10.1161/CIR.0000000000000350 (2016).

2. Lloyd-Jones, D. M., Camargo, C. A., Allen, L. A., Giugliano, R. P. \& O’Donnell, C. J. Predictors of long-term mortality after hospitalization for primary unstable angina pectoris and non-ST-elevation myocardial infarction. The American journal of cardiology 92, 1155-1159 (2003)

3. Fath-Ordoubadi, F. et al. Gender impact on prognosis of acute coronary syndrome patients treated with drug-eluting stents. The American journal of cardiology 110, 636-642, doi: 10.1016/j.amjcard.2012.04.039 (2012).

4. Falk, E. Pathogenesis of atherosclerosis. J Am Coll Cardiol 47, C7-12, doi: 10.1016/j.jacc.2005.09.068 (2006).

5. Langer, H. F. \& Gawaz, M. Platelet-vessel wall interactions in atherosclerotic disease. Thrombosis and haemostasis 99, 480-486, doi: 10.1160/TH07-11-0685 (2008).

6. Jennings, L. K. Mechanisms of platelet activation: need for new strategies to protect against platelet-mediated atherothrombosis. Thrombosis and haemostasis 102, 248-257, doi: 10.1160/TH09-03-0192 (2009).

7. Frangogiannis, N. G., Smith, C. W. \& Entman, M. L. The inflammatory response in myocardial infarction. Cardiovascular research 53, 31-47 (2002).

8. Zhou, D. et al. Platelet-to-Lymphocyte Ratio Improves the Predictive Power of GRACE Risk Score for Long-Term Cardiovascular Events in Patients with Acute Coronary Syndrome. Cardiology 134, 39-46, doi: 10.1159/000442939 (2016)

9. Temiz, A. et al. Platelet/lymphocyte ratio and risk of in-hospital mortality in patients with ST-elevated myocardial infarction. Medical science monitor: international medical journal of experimental and clinical research 20, 660-665, doi: 10.12659/MSM.890152 (2014).

10. Ugur, M. et al. The relationship between platelet to lymphocyte ratio and the clinical outcomes in ST elevation myocardial infarction underwent primary coronary intervention. Blood coagulation \& fibrinolysis: an international journal in haemostasis and thrombosis 25, 806-811, doi: 10.1097/MBC.0000000000000150 (2014).

11. Liberati A. et al. The PRISMA statement for reporting systematic reviews and meta-analyses of studies that evaluate healthcare interventions: explanation and elaboration. BMJ 339, b2700 (2009).

12. He, L. P., Tang, X. Y., Ling, W. H., Chen, W. Q. \& Chen, Y. M. Early C-reactive protein in the prediction of long-term outcomes after acute coronary syndromes: a meta-analysis of longitudinal studies. Heart 96, 339-346, doi: 10.1136/hrt.2009.174912 (2010).

13. Zhang, J. \& Yu, K. F. What's the relative risk? A method of correcting the odds ratio in cohort studies of common outcomes. Jama 280, 1690-1691 (1998)

14. Mantel, N. \& Haenszel, W. Statistical aspects of the analysis of data from retrospective studies of disease. Journal of the National Cancer Institute 22, 719-748 (1959).

15. DerSimonian, R. \& Laird, N. Meta-analysis in clinical trials. Controlled clinical trials 7, 177-188 (1986).

16. Egger, M., Smith, G. D. \& Phillips, A. N. Meta-analysis: principles and procedures. BMJ (Clinical research ed.) 315, 1533-1537 (1997).

17. Toprak, C. et al. Platelet/lymphocyte ratio was associated with impaired myocardial perfusion and both in-hospital and long-term adverse outcome in patients with ST-segment elevation acute myocardial infarction undergoing primary coronary intervention. Postepy $w$ kardiologii interwencyjnej=Advances in interventional cardiology 11,288-297, doi: 10.5114/pwki.2015.55599 (2015).

18. Ozcan Cetin, E. H. et al. Platelet to Lymphocyte Ratio as a Prognostic Marker of In-Hospital and Long-Term Major Adverse Cardiovascular Events in ST-Segment Elevation Myocardial Infarction. Angiology 67, 336-345, doi: 10.1177/0003319715591751 (2016).

19. Oylumlu, M. et al. Platelet-to-lymphocyte ratio is a predictor of in-hospital mortality patients with acute coronary syndrome. Anatolian journal of cardiology 15, 277-283, doi: 10.5152/akd.2014.5366 (2015).

20. Kurtul, A. et al. Association of platelet-to-lymphocyte ratio with severity and complexity of coronary artery disease in patients with acute coronary syndromes. The American journal of cardiology 114, 972-978, doi: 10.1016/j.amjcard.2014.07.005 (2014). 
21. Hudzik, B. et al. Platelet-to-lymphocyte ratio is a marker of poor prognosis in patients with diabetes mellitus and ST-elevation myocardial infarction. Biomarkers in medicine 9, 199-207, doi: 10.2217/bmm.14.100 (2015).

22. Azab, B., Shah, N., Akerman, M. \& McGinn, J. T. Jr. Value of platelet/lymphocyte ratio as a predictor of all-cause mortality after nonST-elevation myocardial infarction. Journal of thrombosis and thrombolysis 34, 326-334, doi: 10.1007/s11239-012-0718-6 (2012).

23. Ayca, B. et al. Platelet to lymphocyte ratio as a prognostic marker in primary percutaneous coronary intervention. Platelets 26, 638-644, doi: 10.3109/09537104.2014.968117 (2015)

24. Smith, R. A. et al. The platelet-lymphocyte ratio improves the predictive value of serum CA19-9 levels in determining patient selection for staging laparoscopy in suspected periampullary cancer. Surgery 143, 658-666, doi: 10.1016/j.surg.2007.12.014 (2008).

25. Smith, R. A. et al. Prognosis of resected ampullary adenocarcinoma by preoperative serum CA19-9 levels and platelet-lymphocyte ratio. Journal of gastrointestinal surgery: official journal of the Society for Surgery of the Alimentary Tract 12, 1422-1428, doi: 10.1007/ s11605-008-0554-3 (2008).

26. Dutta, S., Crumley, A. B., Fullarton, G. M., Horgan, P. G. \& McMillan, D. C. Comparison of the prognostic value of tumour- and patient-related factors in patients undergoing potentially curative resection of oesophageal cancer. World journal of surgery 35, 1861-1866, doi: 10.1007/s00268-011-1130-7 (2011).

27. Aliustaoglu, M. et al. The effect of peripheral blood values on prognosis of patients with locally advanced gastric cancer before treatment. Medical oncology 27, 1060-1065, doi: 10.1007/s12032-009-9335-4 (2010).

28. Acet, H. et al. Relationship Between Hematologic Indices and Global Registry of Acute Coronary Events Risk Score in Patients With ST-Segment Elevation Myocardial Infarction. Clinical and applied thrombosis/hemostasis: official journal of the International Academy of Clinical and Applied Thrombosis/Hemostasis 22, 60-68, doi: 10.1177/1076029614533145 (2016).

29. Kurtul, A. et al. Usefulness of the platelet-to-lymphocyte ratio in predicting angiographic reflow after primary percutaneous coronary intervention in patients with acute ST-segment elevation myocardial infarction. The American journal of cardiology 114, 342-347, doi: 10.1016/j.amjcard.2014.04.045 (2014).

30. Li, J. et al. GWAS of blood cell traits identifies novel associated loci and epistatic interactions in Caucasian and African-American children. Human molecular genetics 22, 1457-1464, doi: 10.1093/hmg/dds534 (2013).

31. Gu, X. et al. Prognostic value of platelet to lymphocyte ratio in non-small cell lung cancer: evidence from 3,430 patients. Scientific reports 6, 23893, doi: 10.1038/srep23893 (2016).

32. Curwen, M. Excess winter mortality: a British phenomenon? Health Trends 22, 169-175 (1990).

33. Borowicz-Bienkowska, S. et al. The impact of short-term cardiac rehabilitation on changing dietary habits in patients after acute coronary syndrome. Journal of cardiopulmonary rehabilitation and prevention 33, 234-238, doi: 10.1097/HCR.0b013e318293b47b (2013).

34. Burstein, S. A., Mei, R. L., Henthorn, J., Friese, P. \& Turner, K. Leukemia inhibitory factor and interleukin-11 promote maturation of murine and human megakaryocytes in vitro. Journal of cellular physiology 153, 305-312, doi: 10.1002/jcp.1041530210 (1992).

35. Gawaz, M., Langer, H. \& May, A. E. Platelets in inflammation and atherogenesis. The Journal of clinical investigation 115, 3378-3384, doi: $10.1172 /$ JCI27196 (2005)

36. Lindemann, S., Kramer, B., Seizer, P. \& Gawaz, M. Platelets, inflammation and atherosclerosis. Journal of thrombosis and haemostasis: JTH 5 Suppl 1, 203-211, doi: 10.1111/j.1538-7836.2007.02517.x (2007).

37. Furman, M. I. et al. Increased platelet reactivity and circulating monocyte-platelet aggregates in patients with stable coronary artery disease. J Am Coll Cardiol 31, 352-358 (1998).

38. Tsiara, S., Elisaf, M., Jagroop, I. A. \& Mikhailidis, D. P. Platelets as predictors of vascular risk: is there a practical index of platelet activity? Clinical and applied thrombosis/hemostasis: official journal of the International Academy of Clinical and Applied Thrombosis/ Hemostasis 9, 177-190 (2003).

39. Gary, T. et al. Platelet-to-lymphocyte ratio: a novel marker for critical limb ischemia in peripheral arterial occlusive disease patients. PloS One 8, e67688, doi: 10.1371/journal.pone.0067688 (2013).

40. Thomson, S. P., McMahon, L. J. \& Nugent, C. A. Endogenous cortisol: a regulator of the number of lymphocytes in peripheral blood. Clinical immunology and immunopathology 17, 506-514 (1980).

41. Roy, D. et al. A comparative study of markers of inflammation for the assessment of cardiovascular risk in patients presenting to the emergency department with acute chest pain suggestive of acute coronary syndrome. International journal of cardiology 109, 317-321, doi: 10.1016/j.ijcard.2005.06.030 (2006).

42. Zouridakis, E. G., Garcia-Moll, X. \& Kaski, J. C. Usefulness of the blood lymphocyte count in predicting recurrent instability and death in patients with unstable angina pectoris. The American journal of cardiology 86, 449-451 (2000).

43. Ommen, S. R., Gibbons, R. J., Hodge, D. O. \& Thomson, S. P. Usefulness of the lymphocyte concentration as a prognostic marker in coronary artery disease. The American journal of cardiology 79, 812-814 (1997).

44. Ommen, S. R. et al. Predictive power of the relative lymphocyte concentration in patients with advanced heart failure. Circulation 97, 19-22 (1998)

45. Weng, T. P., Fu, T. C., Wang, C. H., Hsu, C. C. \& Wang, J. S. Activation of lymphocyte autophagy/apoptosis reflects haemodynamic inefficiency and functional aerobic impairment in patients with heart failure. Clinical science 127, 589-602, doi: 10.1042/ CS20130789 (2014).

46. Ayhan, E. et al. Patients with anemia on admission who have undergone primary angioplasty for ST elevation myocardial infarction: in-hospital and long-term clinical outcomes. Coronary artery disease 22, 375-379, doi: 10.1097/MCA.0b013e3283472ac5 (2011).

47. Planer, D. et al. Impact of hyperglycemia in patients with ST-segment elevation myocardial infarction undergoing percutaneous coronary intervention: the HORIZONS-AMI trial. International journal of cardiology 167, 2572-2579, doi: 10.1016/j. ijcard.2012.06.054 (2013).

48. Sen, N. et al. The relationship between admission hemoglobin level and left ventricular systolic functions in patients with first STsegment elevated myocardial infarction. Turk Kardiyoloji Dernegi arsivi: Turk Kardiyoloji Derneginin yayin organidir 38, 233-238 (2010).

49. Schiariti, M., Iannetta, L., Torromeo, C., De Gregorio, M. \& Puddu P. E. Prognostic significance of post percutaneous coronary intervention thrombocytopenia. World J Meta-Anal 2, 24-28 (2014).

50. Schiariti, M. et al. Decline in platelet count and long-term post-PCI ischemic events: implication of the intra-aortic balloon pump. Vascular pharmacology 60, 25-31, doi: 10.1016/j.vph.2013.11.002 (2014).

51. Froom, P. Blood viscosity and the risk of death from coronary heart disease. European heart journal 21, 513-514 (2000).

52. Puddu, P. E. et al. Red blood cells count in short-term prediction of cardiovascular disease incidence in the Gubbio population Study. Acta Cardiol 57, 177-185, (2002).

53. Rousseau, M. et al. Relation between hemoglobin level and recurrent myocardial ischemia in acute coronary syndromes detected by continuous electrocardiographic monitoring. Am J Cardiol 106, 1417-1422 (2010).

54. Kruk, M. et al. Clustering of admission hyperglycemia, impaired renal function and anemia and its impact on in-hospital outcomes in patients with ST-elevation myocardial infarction. Atherosclerosis 209, 558-564, doi: 10.1016/j.atherosclerosis.2009.09.074 (2010).

55. Puddu, P. E. et al. The role of Glycoprotein IIb/IIIa inhibitors in acute coronary syndromes and the interference with anemia. Int $J$ Cardiol, doi: 10.1016/j.ijcard.2016.07.207. (2016).

56. Sterne, J. A., Gavaghan, D. \& Egger, M. Publication and related bias in meta-analysis: power of statistical tests and prevalence in the literature. Journal of clinical epidemiology 53, 1119-1129 (2000).

57. Hoaglin, D. C. Misunderstandings about Q and 'Cochran's Q test' in meta-analysis. Statistics in medicine 35, 485-495, doi: 10.1002/ sim.6632 (2016). 


\section{Author Contributions}

Conceived and designed the study: W.Z.L. and Q.Q.L.; Analyzed the data: W.Z.L., Q.Q.L. and Y.T.; Wrote the manuscript: W.Z.L. and Q.Q.L.

\section{Additional Information}

Competing financial interests: The authors declare no competing financial interests.

How to cite this article: Li, W. et al. Platelet to lymphocyte ratio in the prediction of adverse outcomes after acute coronary syndrome: a meta-analysis. Sci. Rep. 7, 40426; doi: 10.1038/srep40426 (2017).

Publisher's note: Springer Nature remains neutral with regard to jurisdictional claims in published maps and institutional affiliations.

(c) (i) This work is licensed under a Creative Commons Attribution 4.0 International License. The images or other third party material in this article are included in the article's Creative Commons license, unless indicated otherwise in the credit line; if the material is not included under the Creative Commons license, users will need to obtain permission from the license holder to reproduce the material. To view a copy of this license, visit http://creativecommons.org/licenses/by/4.0/

(c) The Author(s) 2017 